\title{
APPROXIMATE SOLUTIONS OF THE GENERALIZED GOŁA̧B-SCHINZEL EQUATION
}

\author{
JACEK CHUDZIAK
}

Received 24 March 2006; Revised 12 July 2006; Accepted 28 July 2006

Motivated by the problem of R. Ger, we show that the generalized Gołąb-Schinzel equation is superstable in the class of functions hemicontinuous at the origin. As a consequence, we obtain the form of approximate solutions of that equation.

Copyright ( 2006 Jacek Chudziak. This is an open access article distributed under the Creative Commons Attribution License, which permits unrestricted use, distribution, and reproduction in any medium, provided the original work is properly cited.

\section{Introduction}

The Gołąb-Schinzel equation

$$
f(x+f(x) y)=f(x) f(y)
$$

and its generalized version

$$
f\left(x+f(x)^{k} y\right)=\lambda f(x) f(y)
$$

play a significant role in the theory of functional equations. Some information on the applications of (1.1) and (1.2) in the determination of substructures of algebraical structures, in the theory of geometric objects and classification of near-rings and quasialgebras, can be found, for example, in [1-5] and in the recent survey paper [6].

At the 38th International Symposium on Functional Equations (2000, Noszvaj, Hungary), R. Ger raised, among others, the problem of Hyers-Ulam stability for the GołąbSchinzel-type functional equations (see [6, page 21] and [12]). In the case of (1.1), this problem has been studied in $[7,8,10]$. Recently, in [9] it has been proved that $(1.2)$ is superstable in the class of continuous functions $f: \mathbb{R} \rightarrow \mathbb{R}$. In the present paper, we deal with the stability problem for (1.2) in the case where $f$ is defined on a linear space over the field $K$ of real or complex numbers and takes its values in $K$.

Throughout the paper, $\mathbb{N}, \mathbb{Z}$, and $\mathbb{R}$ stand for the sets of all positive integers, integers, and real numbers, respectively. 
2 Approximate solutions of the Gołąb-Schinzel equation

\section{Results}

From now on we assume that $K$ is a field of real or complex numbers, $X$ is a vector space over $K$ and $k \in \mathbb{N}, \lambda \in K \backslash\{0\}$ and $\varepsilon \geq 0$ are fixed. We begin with some remarks concerning bounded solutions of the inequality

$$
\left|f\left(x+f(x)^{k} y\right)-\lambda f(x) f(y)\right| \leq \varepsilon \quad \text { for } x, y \in X .
$$

Remark 2.1. A straightforward calculation shows that every function $f: X \rightarrow K$ such that $|f(x)| \leq \min \{1 /|\lambda|, \varepsilon / 2\}$ for $x \in X$ satisfies (2.1).

Remark 2.2. If $f: X \rightarrow K$ is an unbounded function satisfying (2.1), then $f(0)=1 / \lambda$. Otherwise, setting in (2.1) $y=0$, we would have

$$
|f(x)| \leq \frac{\varepsilon}{|1-\lambda f(0)|} \quad \text { for } x \in X
$$

Remark 2.3. If $f: X \rightarrow K$ is a bounded function satisfying (2.1), then

$$
|f(x)| \leq \frac{1+\sqrt{1+4|\lambda| \varepsilon}}{2|\lambda|} \text { for } x \in X
$$

In fact, suppose that $f: X \rightarrow K$ is a bounded function satisfying (2.1) and

$$
M:=\sup \{|f(x)|: x \in \mathbb{R}\}>\frac{1+\sqrt{1+4|\lambda| \varepsilon}}{2|\lambda|}
$$

Then

$$
|\lambda| M^{2}-M-\varepsilon>0 .
$$

Therefore, taking a sequence $\left(x_{n}: n \in \mathbb{N}\right)$ of elements of $X$ such that $\lim _{n \rightarrow \infty}\left|f\left(x_{n}\right)\right|=M$, we obtain

$$
\lambda^{2} f\left(x_{n}\right)^{2}>\lambda^{2} M^{2}-|\lambda| \varepsilon>|\lambda| M
$$

for sufficiently large $n \in \mathbb{N}$. Since

$$
\lambda f\left(x_{n}+f\left(x_{n}\right)^{k} x_{n}\right) \leq|\lambda| M \quad \text { for } n \in \mathbb{N},
$$

this yields that

$$
\left|\lambda f\left(x_{n}+f\left(x_{n}\right)^{k} x_{n}\right)-\lambda^{2} f\left(x_{n}\right)^{2}\right| \geq \lambda^{2} f\left(x_{n}\right)^{2}-|\lambda| M
$$

for sufficiently large $n \in \mathbb{N}$. Furthermore, by (2.5),

$$
\lim _{n \rightarrow \infty}\left(\lambda^{2} f\left(x_{n}\right)^{2}-|\lambda| M\right)=\lambda^{2} M^{2}-|\lambda| M>|\lambda| \varepsilon
$$


Consequently,

$$
\left|\lambda f\left(x_{n}+f\left(x_{n}\right)^{k} x_{n}\right)-\lambda^{2} f\left(x_{n}\right)^{2}\right|>|\lambda| \varepsilon
$$

for sufficiently large $n \in \mathbb{N}$, which contradicts (2.1).

To formulate the main result of the paper, we need the following definition (cf. [13, page 427]).

Definition 2.4. A function $f: X \rightarrow K$ is hemicontinuous at the origin provided, for every $x \in X$, the function $f_{x}: K \rightarrow K$, given by

$$
f_{x}(t)=f(t x) \quad \text { for } t \in K,
$$

is continuous at 0 .

Theorem 2.5. If $f: X \rightarrow K$ is a hemicontinuous at the origin function satisfying (2.1), then either (2.3) holds, or

$$
f\left(x+f(x)^{k} y\right)=\lambda f(x) f(y) \quad \text { for } x, y \in X .
$$

Proof. Assume that a function $f: X \rightarrow K$ is hemicontinuous at the origin and satisfies (2.1). In view of Remark 2.3, it is enough to consider the case, where $f$ is unbounded. Then, according to Remark 2.2, $f(0)=1 / \lambda$. Thus, setting in (2.1) $x=0$ and replacing $y$ by $\lambda^{k} y$, we obtain

$$
\left|f\left(\lambda^{k} y\right)-f(y)\right| \leq \varepsilon \quad \text { for } y \in X .
$$

Further, inserting in (2.1) $(y-x) / f(x)^{k}$ in place of $y$, we get

$$
\left|f(y)-\lambda f(x) f\left(\frac{y-x}{f(x)^{k}}\right)\right| \leq \varepsilon \quad \text { for } x \in X \backslash f^{-1}\{0\}, y \in X .
$$

Take a sequence $\left(x_{n}: n \in \mathbb{N}\right)$ of elements of $X \backslash f^{-1}(\{0\})$ such that

$$
\lim _{n \rightarrow \infty}\left|f\left(x_{n}\right)\right|=\infty .
$$

For $x, y \in X$ and $n \in \mathbb{N}$, define

$$
\begin{gathered}
c_{n}(x, y):=\lambda^{k} x_{n}+\lambda^{k} f\left(x_{n}\right)^{k} x+f\left(\lambda^{k}\left(x_{n}+f\left(x_{n}\right)^{k} x\right)\right)^{k} y, \\
d_{n}(x, y):=\lambda^{k}\left(x_{n}+f\left(x_{n}\right)^{k} x+f\left(x_{n}\right)^{k} f(x)^{k} y\right) .
\end{gathered}
$$

Then, for every $x, y \in X$ and $n \in \mathbb{N}$, we have

$$
\begin{aligned}
& f\left(d_{n}(x, y)\right)-\lambda f\left(x_{n}\right) f\left(x+f(x)^{k} y\right) \\
& =f\left(\lambda^{k}\left(x_{n}+f\left(x_{n}\right)^{k} x+f\left(x_{n}\right)^{k} f(x)^{k} y\right)\right)-f\left(x_{n}+f\left(x_{n}\right)^{k} x+f\left(x_{n}\right)^{k} f(x)^{k} y\right) \\
& \quad+f\left(x_{n}+f\left(x_{n}\right)^{k}\left(x+f(x)^{k} y\right)\right)-\lambda f\left(x_{n}\right) f\left(x+f(x)^{k} y\right) .
\end{aligned}
$$


4 Approximate solutions of the Gołąb-Schinzel equation

Note that in view of (2.1) and (2.13), for every $x, y \in X$, the right-hand side of the last equality is bounded. Thus

$$
\lim _{n \rightarrow \infty} \frac{f\left(d_{n}(x, y)\right)}{\lambda f\left(x_{n}\right)}=f\left(x+f(x)^{k} y\right) \quad \text { for } x, y \in X .
$$

Next, by (2.1) and (2.13), we get

$$
\lambda f(x)=\lim _{n \rightarrow \infty} \frac{f\left(x_{n}+f\left(x_{n}\right)^{k} x\right)}{f\left(x_{n}\right)}=\lim _{n \rightarrow \infty} \frac{f\left(\lambda^{k}\left(x_{n}+f\left(x_{n}\right)^{k} x\right)\right)}{f\left(x_{n}\right)} \text { for } x \in X .
$$

Hence

$$
\lim _{n \rightarrow \infty}\left|f\left(\lambda^{k}\left(x_{n}+f\left(x_{n}\right)^{k} x\right)\right)\right|=\infty \quad \text { for } x \in X \backslash f^{-1}(\{0\}) .
$$

Since, in view of (2.1), for every $x, y \in X$ and $n \in \mathbb{N}$, we have

$$
\begin{aligned}
& \left|f\left(c_{n}(x, y)\right)-\lambda f\left(\lambda^{k}\left(x_{n}+f\left(x_{n}\right)^{k} x\right)\right) f(y)\right| \\
& \quad=\left|f\left(\lambda^{k}\left(x_{n}+f\left(x_{n}\right)^{k} x\right)+f\left(\lambda^{k}\left(X_{n}+f\left(x_{n}\right)^{k} x\right)\right)^{k} y\right)-\lambda f\left(\lambda^{k}\left(x_{n}+f\left(x_{n}\right)^{k} x\right)\right) f(y)\right| \leq \varepsilon,
\end{aligned}
$$

this yields that

$$
\lambda f(y)=\lim _{n \rightarrow \infty} \frac{f\left(c_{n}(x, y)\right)}{f\left(\lambda^{k}\left(x_{n}+f\left(x_{n}\right)^{k} x\right)\right)} \quad \text { for } x \in X \backslash f^{-1}(\{0\}), y \in X .
$$

Thus, taking into account (2.19), we get

$$
\lim _{n \rightarrow \infty} \frac{f\left(c_{n}(x, y)\right)}{f\left(x_{n}\right)}=\lambda^{2} f(x) f(y) \quad \text { for } x \in X \backslash f^{-1}(\{0\}), y \in X .
$$

Hence, for every $x, y \in X \backslash f^{-1}(\{0\})$, we have

$$
c_{n}(x, y) \in X \backslash f^{-1}(\{0\}),
$$

so, in view of (2.14),

$$
\lim _{n \rightarrow \infty} \frac{f\left(d_{n}(x, y)\right)-\lambda f\left(c_{n}(x, y)\right) f\left(\left(d_{n}(x, y)-c_{n}(x, y)\right) / f\left(c_{n}(x, y)\right)^{k}\right)}{\lambda f\left(x_{n}\right)}=0 .
$$

Therefore, taking into account (2.18) and (2.23), for every $x, y \in X \backslash f^{-1}(\{0\})$, we obtain

$$
f\left(x+f(x)^{k} y\right)=\lambda^{2} f(x) f(y) \lim _{n \rightarrow \infty} f\left(\frac{d_{n}(x, y)-c_{n}(x, y)}{f\left(c_{n}(x, y)\right)^{k}}\right) .
$$


Moreover, for every $x, y \in X \backslash f^{-1}(\{0\})$, we have

$$
\frac{d_{n}(x, y)-c_{n}(x, y)}{f\left(c_{n}(x, y)\right)^{k}}=\frac{\lambda^{k} f\left(x_{n}\right)^{k} f(x)^{k}-f\left(\lambda^{k}\left(x_{n}+f\left(x_{n}\right)^{k} x\right)\right)^{k}}{f\left(c_{n}(x, y)\right)^{k}} y
$$

and, by (2.19) and (2.23),

$$
\begin{aligned}
\lim _{n \rightarrow \infty} & \frac{\lambda^{k} f\left(x_{n}\right)^{k} f(x)^{k}-f\left(\lambda^{k}\left(x_{n}+f\left(x_{n}\right)^{k} x\right)\right)^{k}}{f\left(\left(c_{n} x, y\right)\right)^{k}} \\
\quad=\lim _{n \rightarrow \infty}\left(\frac{f\left(x_{n}\right)}{f\left(c_{n}(x, y)\right)}\right)^{k}\left(\lambda^{k} f(x)^{k}-\left(\frac{f\left(\lambda^{k}\left(x_{n}+f\left(x_{n}\right)^{k} x\right)\right)}{f\left(x_{n}\right)}\right)\right. & =0
\end{aligned}
$$

Thus, as $f$ is hemicontinuous at the origin, we get

$$
\lim _{n \rightarrow \infty} f\left(\frac{d_{n}(x, y)-c_{n}(x, y)}{f\left(c_{n}(x, y)\right)^{k}}\right)=f_{y}(0)=f(0)=\frac{1}{\lambda} .
$$

Hence, in view of (2.26), we obtain

$$
f\left(x+f(x)^{k} y\right)=\lambda f(x) f(y) \quad \text { for } x, y \in X \backslash f^{-1}(\{0\}) .
$$

Next, taking in (2.30) $x=0$ and replacing $y$ by $\lambda^{k} y$, we get

$$
f\left(\lambda^{k} y\right)=f(y) \quad \text { for } y \in X \backslash f^{-1}(\{0\}) .
$$

Since, by (2.19), for every $x \in X \backslash f^{-1}(\{0\})$ and $n \in \mathbb{N}$,

$$
x_{n}+f\left(x_{n}\right)^{k} x \in X \backslash f^{-1}(\{0\}),
$$

making use of (2.30) and (2.31), we conclude that

$$
f\left(\lambda^{k}\left(x_{n}+f\left(x_{n}\right)^{k} x\right)\right)=f\left(x_{n}+f\left(x_{n}\right)^{k} x\right)=\lambda f\left(x_{n}\right) f(x) .
$$

Thus

$$
c_{n}(x, y)=d_{n}(x, y) \quad \text { for } x \in X \backslash f^{-1}(\{0\}), y \in X, n \in \mathbb{N} \text {, }
$$

whence taking into account (2.18) and (2.23), we get

$$
f\left(x+f(x)^{k} y\right)=\lambda f(x) f(y) \quad \text { for } x \in X \backslash f^{-1}(\{0\}), y \in X .
$$

As for $x \in f^{-1}(\{0\}),(2.12)$ trivially holds, this completes the proof.

Remark 2.6. From Theorem 2.5, it follows that (1.2) is superstable in the class of functions $f: X \rightarrow K$ hemicontinuous at the origin. For more information concerning superstability we refer to [11, Chapter 4] and [14, Chapter 5]. 
Remark 2.7. Theorem 2.5 remains true, if instead of the hemicontinuity at the origin, we assume that for every $x \in X \backslash f^{-1}(\{0\})$, there exists a limit (not necessarily finite) $l(x):=\lim _{t \rightarrow 0} f(t x)$. In such a case from $(2.26)$ we derive that

$$
f\left(x+f(x)^{k} y\right)=\lambda^{2} f(x) f(y) l(y) \quad \text { for } x, y \in X \backslash f^{-1}(\{0\}) .
$$

Thus, in view of (2.19), we obtain

$$
l(y)=\frac{1}{\lambda^{2} f(y)} \lim _{n \rightarrow \infty} \frac{f\left(x_{n}+f\left(x_{n}\right)^{k} y\right)}{f\left(x_{n}\right)}=\frac{1}{\lambda} \quad \text { for } y \in X \backslash f^{-1}(\{0\}) .
$$

Consequently, (2.36) becomes (2.30) and, arguing as previously, we get the assertion.

Now, applying Theorem 2.5, we obtain the following results, which generalize to some extend [9, Propositions 1-3].

Proposition 2.8. If $\left|\lambda^{k}\right| \neq 1$, then every hemicontinuous at the origin function $f: X \rightarrow K$ satisfying (2.1) is bounded.

Proof. Suppose that $\left|\lambda^{k}\right| \neq 1$ and $f: X \rightarrow K$ is an unbounded function hemicontinuous at the origin and satisfies (2.1). Then, according to Theorem 2.5, (2.12) is valid. Thus, taking in (2.12) $x=0$ and using Remark 2.2, we get $f\left(\lambda^{-k} y\right)=f(y)$ for $y \in X$, whence by induction

$$
f\left(\lambda^{n k} y\right)=f(y) \quad \text { for } y \in X, n \in \mathbb{Z}
$$

As $f$ is hemicontinuous at the origin, letting in the last equality $n \rightarrow \infty$, whenever $\left|\lambda^{k}\right|<1$; and $n \rightarrow-\infty$ otherwise, we obtain

$$
f(y)=f_{y}(0)=f(0)=\frac{1}{\lambda} \quad \text { for } y \in X .
$$

Hence $f$ is constant, which yields a contradiction and completes the proof.

Proposition 2.9. Assume that $\lambda=1$ and $f: X \rightarrow K$ is an unbounded function hemicontinuous at the origin. Then $f$ satisfies (2.1) if and only if $f(X) \subset \mathbb{R}$ or $k=1$, and

(i) if $f(X) \subset \mathbb{R}$, then there exists a nontrivial $\mathbb{R}$-linear functional $L: X \rightarrow \mathbb{R}$ such that

$\left(1^{\circ}\right)$ in the case where $k$ is an odd number,

$$
f(x)=(L(x)+1)^{1 / k} \quad \text { for } x \in X
$$

or

$$
f(x)=(\max \{L(x)+1,0\})^{1 / k} \quad \text { for } x \in X ;
$$

$\left(2^{\circ}\right)$ in the case where $k$ is even, $f$ is of the form (2.41);

(ii) if $f(X) \backslash \mathbb{R} \neq \varnothing$ and $k=1$, then there exists a nontrivial $\mathbb{C}$-linear functional $L: X \rightarrow$ $\mathbb{C}$ such that

$$
f(x)=L(x)+1 \quad \text { for } x \in X
$$


Proof. It is easy to check that each of conditions (i) and (ii) implies (2.1). So, assume that $f$ satisfies (2.1). Then, by Theorem 2.5,

$$
f\left(x+f(x)^{k} y\right)=f(x) f(y) \quad \text { for } x, y \in X .
$$

Further, in view of Remark 2.2, $f(0)=1$. Since $f$ is hemicontinuous at the origin, this means that the origin is an algebraically interior point of the set $X \backslash f^{-1}(\{0\})$ (see [4, Definition 1]). Thus, applying [4, Theorem 3], we get the assertion.

Proposition 2.10. Assume that $\lambda=-1$.

(i) If $k$ is an odd number, then every hemicontinuous at the origin function $f: X \rightarrow K$ satisfying (2.1) is bounded.

(ii) If $k$ is even, then an unbounded and hemicontinuous at the origin function $f: X \rightarrow K$ satisfies (2.1) if and only if there exists a nontrivial $\mathbb{R}$-linear functional $L: X \rightarrow \mathbb{R}$ such that

$$
f(x)=-(\max \{L(x)+1,0\})^{1 / k} \quad \text { for } x \in X .
$$

Proof. Suppose that $f: X \rightarrow K$ is an unbounded and hemicontinuous at the origin function satisfying (2.1). Then, applying Theorem 2.5 and Remark 2.2, we get

$$
f\left(x+f(x)^{k} y\right)=-f(x) f(y) \quad \text { for } x, y \in X
$$

and $f(0)=-1$, respectively. Therefore, if $k$ is an odd number, then making use of (2.45) with $x=0$, we obtain that $f$ is an even function. Hence, replacing in (2.45) $y$ by $-y$, we conclude that a function $\bar{f}:=-f$ satisfies (2.43). So, arguing as in the proof of Proposition 2.9, we obtain that one of the conditions (i) and (ii) of Proposition 2.9 is valid for $\bar{f}$. As $\bar{f}$ is even, this gives a contradiction.

Now, assume that $k$ is an even number. Then from (2.45) we deduce again that $\bar{f}:=$ $-f$ satisfies (2.43) (however, contrary to the previous case, $\bar{f}$ need not be even). Thus, repeating the arguments from the proof of Proposition 2.9, we obtain that there exists a nontrivial $\mathbb{R}$-linear functional $L: X \rightarrow \mathbb{R}$ such that

$$
\bar{f}(x)=(\max \{L(x)+1,0\})^{1 / k} \quad \text { for } x \in X .
$$

This implies (2.44). Since the converse is easy to check, the proof is completed.

\section{References}

[1] J. Aczél and J. Dhombres, Functional Equations in Several Variables, Encyclopedia of Mathematics and Its Applications, vol. 31, Cambridge University Press, Cambridge, 1989.

[2] N. Brillouët-Belluot, On some functional equations of Gołab-Schinzel type, Aequationes Mathematicae 42 (1991), no. 2-3, 239-270.

[3] N. Brillouët-Belluot and J. Dhombres, Équations fonctionnelles et recherche de sous-groupes, Aequationes Mathematicae 31 (1986), no. 2-3, 253-293.

[4] J. Brzdęk, Subgroups of the group $Z_{n}$ and a generalization of the Gołab-Schinzel functional equation, Aequationes Mathematicae 43 (1992), no. 1, 59-71.

[5] Some remarks on solutions of the functional equation $f\left(x+f(x)^{n} y\right)=t f(x) f(y)$, Publicationes Mathematicae Debrecen 43 (1993), no. 1-2, 147-160. 


\section{Approximate solutions of the Gołąb-Schinzel equation}

[6] _ The Golab-Schinzel equation and its generalizations, Aequationes Mathematicae 70 (2005), no. 1-2, 14-24.

[7] J. Chudziak, Approximate solutions of the Gołab-Schinzel equation, Journal of Approximation Theory 136 (2005), no. 1, 21-25.

[8] On a functional inequality related to the stability problem for the Gotab-Schinzel equation, Publicationes Mathematicae Debrecen 67 (2005), no. 1-2, 199-208.

[9] _ Stability of the generalized Gołab-Schinzel equation, Acta Mathematica Hungarica 113 (2006), 115-126.

[10] J. Chudziak and J. Tabor, On the stability of the Golab-Schinzel functional equation, Journal of Mathematical Analysis and Applications 302 (2005), no. 1, 196-200.

[11] G. L. Forti, Hyers-Ulam stability of functional equations in several variables, Aequationes Mathematicae 50 (1995), no. 1-2, 143-190.

[12] R. Ger, A collection of problems in stability theory, Report of Meeting, the 38th International Symposium on Functional Equations, Noszvaj, June 2000, Aequationes Mathematicae 61 (2001), no. $3,281-320$.

[13] S. Gudder and D. Strawther, Orthogonally additive and orthogonally increasing functions on vector spaces, Pacific Journal of Mathematics 58 (1975), no. 2, 427-436.

[14] D. H. Hyers, G. Isac, and Th. M. Rassias, Stability of Functional Equations in Several Variables, Progress in Nonlinear Differential Equations and Their Applications, vol. 34, Birkhäuser Boston, Massachusetts, 1998.

Jacek Chudziak: Department of Mathematics, University of Rzeszów, Aleja Rejtana 16 C, Rzeszów 35-959, Poland

E-mail address: chudziak@univ.rzeszow.pl 